\title{
Existence Theorems Concerning Simple Integrals of the Calculus of Variations for Discontinuous Solutions
}

\author{
L. Cesari, P. Brandi \& A. Salvadori \\ Dedicated to James Serrin on his $60^{\text {th }}$ birthday
}

\section{Introduction}

In this paper we apply the direct method of the calculus of variations, based on lower semicontinuity and lower closure (see [5]), to prove the existence of optimal solutions $x(t)=\left(x^{1}, \ldots, x^{n}\right), t_{1} \leqq t \leqq t_{2}$, for which $\alpha$ components $y(t)=\left(x^{1}, \ldots, x^{\alpha}\right)$ are $\mathrm{AC}$ and $n-\alpha$ components $z(t)=\left(x^{\alpha+1}, \ldots, x^{n}\right)$ are BV and not necessarily AC. If $\alpha=0$ all components of $x$ are BV, and in this situation no growth assumption is made on the integrand function. The cost functional $\mathscr{I}$ is of SeRRIN type ([14]), i.e. it is obtained from the usual integral expression $I$ by means of a limit process, based on a topology $\tau$, of this integral $I$ over curves $x_{k}$ whose components are all AC. The topology $\tau$ that we use here is the topology of uniform convergence on the $y_{k}$ components and pointwise convergence almost everywhere on the $z_{k}$ components. This pointwise convergence almost everywhere has been used by CESARI in the study of area of discontinuous surfaces ([4], 1936) and in existence theorems concerning simple integrals for AC solutions (see [5], Chapt. 15 and the papers cited there).

In Section 2 we first prove a closure theorem (Theorem 1) for problems in which mere pointwise convergence almost everywhere is adopted. The closure theorem is used, in Section 3, for proving a lower semicontinuity theorem (Theorem $1^{\prime}$ ) based on the topology $\tau$. The same lower semicontinuity theorem allows us to prove that $\mathscr{I}$ is a true extension of $I$, in the sense that $\mathscr{I}=I$ whenever all components are $\mathrm{AC}$.

In Section 3 we prove also an existence theorem for the absolute minimum of extended problems of the calculus of variations with constraints on the direction of the tangent. In Section 4 we derive, as a corollary, an existence theorem for the absolute minimum of problems of optimal control. For a different viewpoint connecting Serrin-type integrals, usual integrals, and Burkill-Cesari integrals we mention the paper [3] by CANDELORO \& PUCCI, where also lower semicontinuity theorems are given for solutions which are only continuous and of bounded variation.

Elsewhere ([6b]) the present work on discontinuous solutions will be extended 
to multiple integrals of the calculus of variations and functions of $v>1$ independent variables. Therefore, the BV concept in [6b] will be the one introduced by CesARI in 1936 ([4]) and shown by KRICKEBERG ([12]) to be equivalent to the one in terms of distributions. Later the functions of bounded variation in the sense of CESARI were briefly denoted as BVC by CONWAY \& SMOLLER ([8]), DAFERMos ([9]) and DiPerna ([10]). The functions of bounded variation defined in the equivalent terms of distributions were briefly denoted as BV by VOLPERT ([16]) and others. In order that the present work, which concerns functions of one variable, be in harmony with [6b], we use the notations from [6b].

\section{A closure theorem with components converging only pointwise}

Let $A$ be a subset of the $(t, x)$-space $R^{n+1}$ whose projection on the $t$-axis contains the fixed interval $\left[t_{1}, t_{2}\right]$. Let $Q(t, x),(t, x) \in A, Q(t, x) \subset R^{n}$, or $Q: A \rightarrow R^{n}$, be a given set valued function.

Following CESARI [5] we shall say that the set function $Q$ has property $(Q)$ at the point $(\bar{t}, \bar{x})$, with respect to $(t, x)$, if

$$
Q(\bar{t}, \bar{x})=\bigcap_{\delta>0} \mathrm{cl} \mathrm{co} \bigcup_{(t, x) \in B(\bar{t}, \bar{x} ; \delta)} Q(t, x)
$$

where $B(\bar{t}, \bar{x} ; \delta)=\{(t, x) \in A:|(t, x)-(\bar{t}, \bar{x})| \leqq \delta\}$. Let $Q(\bar{t}, \bar{x} ; \delta)=\bigcup Q(t, x)$ for $(t, x) \in B(\bar{t}, \bar{x} ; \delta)$.

Analogously, $Q$ is said to have property $(Q)$ at the point $(\bar{t}, \bar{x})$, with respect to $x$ only, if

$$
Q(\bar{t}, \bar{x})=\bigcap_{\delta>0} \operatorname{clco} \bigcup_{x \in B^{\prime}(\bar{t}, \bar{x} ; \delta)} Q(\bar{t}, x)
$$

where $B^{\prime}(\bar{t}, \bar{x} ; \delta)=\{(t, x) \in A:|x-\bar{x}| \leqq \delta\}$. The corresponding Kuratowski properties (K) are obtained by writing only $\mathrm{cl}$, instead of cl co, in the relations above.

We mention here that a summable function $x(t)$ from $\left[t_{1}, t_{2}\right]$ into $R^{n}$, or $x:\left[t_{1}, t_{2}\right] \rightarrow R^{n}$, is said to be of bounded variation in the sense of Cesari, briefly $\mathrm{BVC}$, if it is equivalent to a BV function $\tilde{x}:\left[t_{1}, t_{2}\right] \rightarrow R^{n}$. It may well occur that $x$ is equivalent to infinitely many BV functions $\tilde{x}$. In this case, at every point $t_{0} \in\left(t_{1}, t_{2}\right)$ of (first kind) discontinuity for $\tilde{x}$, we may take $\tilde{x}\left(t_{0}\right)$ so that $\tilde{x}\left(t_{0}-0\right)$ $\leqq \tilde{x}\left(t_{0}\right) \leqq \tilde{x}\left(t_{0}+0\right)$ or the same relations with the sign $\geqq$. Also, we may take $\tilde{x}\left(t_{1}\right)=\tilde{x}\left(t_{1}+0\right), \tilde{x}\left(t_{2}\right)=\tilde{x}\left(t_{2}-0\right)$. With this choice for $\tilde{x}$ the variation $V(\tilde{x})$ is uniquely determined and it has the minimum value for all $\tilde{x}$ equivalent to $x$. We take, by definition of generalized variation $V^{*}(x)$ the number $V^{*}(x)=$ $V(\tilde{x})$, for $\tilde{x}$ chosen as stated. Moreover we take, by definition $x^{\prime}=\tilde{x}^{\prime}$ (a.e. in $\left.\left[t_{1}, t_{2}\right]\right)$.

Analogously, $x$ is said to be absolutely continuous in the generalized sense, briefly $\mathrm{ACg}$ if $x$ is equivalent to an $\mathrm{AC}$ function $\tilde{x}$. In this case $\tilde{x}$ is uniquely defined, and for the generalized variation we take $V^{*}(x)=V(\tilde{x})$.

For further properties of such functions see [4], [6a], [1], [2], [13]. 
We shall consider the orientor field equation

$$
(t, x(t)) \in A, \quad x^{\prime}(t) \in Q(t, x(t)), \quad \text { a.e. in }\left[t_{1} t_{2}\right] .
$$

that is, the problem of determining a BVC function $x$ satisfying these relations.

We state and prove now a closure theorem which replaces, in the present situation, the closure theorem 15.2.i of [5].

Theorem 1 (A closure theorem). Let us assume that (i) $A$ is closed; (ii) the set valued function $Q$ has closed and convex values; (iii) the set valued function $Q$ has property $(Q)$, with respect to $(t, x)$, at every point $(\bar{t}, \bar{x}) \in A$, with the exception perhaps of a set of points whose $t$-coordinate lies in a set $H$ of measure zero in $\left[t_{1}, t_{2}\right]$.

Let $x_{k}:\left[t_{1}, t_{2}\right] \rightarrow R^{n}, k \in N$, be a sequence of $\mathrm{ACg}$ solutions of the orientor field (3), and assume that $V^{*}\left(x_{k}\right) \leqq V_{0}, k \in N$, and that $x_{k} \rightarrow x$ pointwise a.e. in $\left[t_{1}, t_{2}\right]$, with $x \in \mathrm{BVC}$. Then the function $x$ is a solution of the orientor field relation (3).

Proof. (a) By the hypotheses it follows that

$$
(t, x(t)) \in A, \quad \text { a.e. in }\left[\begin{array}{ll}
t_{1} & t_{2}
\end{array}\right],
$$

so we have only to prove that

$$
x^{\prime}(t) \in Q(t, x(t)) \text { - a.e. in }\left[t_{1}, t_{2}\right] .
$$

In order to see that, without loss of generality we can suppose that $x$ is BV and $x_{k}$ is $\mathrm{AC}, k \in N$.

Let $T_{0} \subset\left[t_{2}, t_{2}\right]$ be a set of measure zero such that in $\left[t_{1}, t_{2}\right]-T_{0}$ we have

$$
\lim _{k \rightarrow \infty} x_{k}(t)=x(t) \quad \text { and } \quad x^{\prime}(t)=x_{a}^{\prime}(t)
$$

where $x=x_{a}+x_{s}$ denotes the Jordan decomposition of $x$.

(b) Now for every $m \in N$, we divide $\left[t_{1}, t_{2}\right]$ into $m$ equal parts $I_{r}^{(m)}, r=$ $1, \ldots, m$, each of length $\left(t_{2}-t_{1}\right) m^{-1}=T m^{-1}$; and denote by $T_{1} \subset\left[t_{1}, t_{2}\right]$ the set of all points of subdivision, so that $T_{1}$ is denumerable and, therefore, has measure zero.

Let $m \in N$ and $\varepsilon>0$ be fixed. For every $k \in N$, we consider those intervals $I_{r}^{(m)}$ if any, such that $\omega\left(x_{k}, I_{r}^{(m)}\right) \geqq \varepsilon$, where $\omega\left(x_{k}, I\right)$ denotes the oscillation of $x_{k}$ over $I$.

Let $S_{k}^{(m)}$ be the system of such intervals, or

$$
S_{k}^{(m)}=\left\{I_{r}^{(m)}, r=1, \ldots, m: \omega\left(x_{k}, I_{r}^{(m)}\right) \geqq \varepsilon\right\} .
$$

We now proceed to the determination of a suitable set $\Sigma^{(m)}$ and to the extraction of a suitable subsequence of $\left(x_{k}\right)_{k \in N}$. First, if $I_{1}^{(m)} \in S_{k}^{(m)}$ for all $k$ sufficiently large, we put $I_{1}^{(m)}$ in $\Sigma^{(m)}$; if not then there are infinitely many $k \in N$ such that $I_{1}^{(m)} \notin S_{k}^{(m)}$ and we denote by $\left(k_{1 s}\right)_{s \in N}$ such sequence, i.e. $I_{1}^{(m)} \notin S_{k_{1 s}}^{(m)}, s \in N$. If $I_{2}^{(m)} \in S_{k_{1 s}}^{(m)}$ for all $s$ sufficiently large, we put $I_{2}^{(m)}$ in $\Sigma^{(m)}$; if not then there 
are infinitely many $s \in N$ such that $I_{2}^{(m)} \notin S_{k_{1 s}}^{(m)}$. We denote such a sequence by $\left(k_{2 s}\right)_{s \in N}$; then $\left(k_{2 s}\right) \subset\left(k_{1 s}\right)$ and $I_{2}^{(m)} \notin S_{k_{2 s}}^{(m)}, s \in N$.

We proceed as indicated for $I_{3}^{(m)}, \ldots, I_{m}^{(m)}$.

At the end we have a set $\Sigma^{(m)}$ made up of all points of certain intervals $I_{r}^{(m)}$, $r=1, \ldots, m$, say, for simplicity $\Sigma^{(m)}=\left\{I_{i}^{(m)}, i=1, \ldots, v\right\}$, and a final sequence $\left(k_{m s}\right)_{s \in N}$ with $\left(k_{m s}\right)_{s \in N} \subset\left(k_{m-1, s}\right)_{s \in N} \subset \ldots \subset\left(k_{1 s}\right)_{s \in N}$.

Note that for all $k_{m s}$ sufficiently large we have

$$
\omega\left(x_{k_{m s}}, I_{i}^{(m)}\right) \geqq \varepsilon, \quad i=1, \ldots, v
$$

and hence

$$
\nu \varepsilon \leqq \sum_{i=1}^{\nu} \omega\left(x_{k_{m s}}, I_{i}^{(m)}\right) \leqq \sum_{r=1}^{m} \omega\left(x_{k_{m s}}, I_{r}^{(m)}\right) \leqq V\left(x_{k_{m s}}\right) \leqq V_{0},
$$

i.e. $\nu \leqq V_{\mathrm{o}} \varepsilon^{-1}$.

This implies that

$$
\text { meas }\left(\Sigma^{(m)}\right)=\sum_{i=1}^{v} \text { meas }\left(I_{i}^{(m)}\right)=\nu T m^{-1} \leqq V_{0} T / \varepsilon m .
$$

Hence, for every $\varepsilon>0$ we can choose an integer $m_{\varepsilon}$ sufficiently large that

$$
\text { meas }\left(\Sigma^{\left(m_{\varepsilon}\right)}\right) \leqq V_{0} T / \varepsilon m_{\varepsilon}<\varepsilon .
$$

Now we take $\varepsilon$ ranging in succession over the values $\left(1 / 2^{\lambda}\right)_{\lambda \in N}$. Thus, for $\lambda=1$ then $\varepsilon=1 / 2$ and, starting from the original sequence $(k)_{k \in N}$, we obtain from the above an integer $m_{\varepsilon}$, which we denote by $m_{1}$, a set $\Sigma^{\left(m_{\varepsilon}\right)}$, which we denote by $\Sigma^{(1)}$, and a subsequence $\left(k_{m s}\right)_{s \in N}$ that we denote by $\left(k_{s}^{1}\right)_{s \in N}$.

For $\lambda=2$ then $\varepsilon=1 / 2^{2}$ and, starting from the sequence $\left(k_{s}^{1}\right)_{s \in N}$ we obtain, as before, an integer $m_{2}$, a set $\Sigma^{(2)}$ and a sequence $\left(k_{s}^{2}\right)_{s \in N}$.

Proceeding as indicated for the generic $\lambda \in N$, we see that $\varepsilon=1 / 2^{\lambda}$ and, starting from the sequence $\left(k_{s}^{\lambda-1}\right)_{s \in N}$, we obtain an integer $m_{\lambda}$, a set $\Sigma^{(\lambda)}$ and a subsequence $\left(k_{s}^{\lambda}\right)_{s \in N}$ as before.

It is not restrictive to assume that $\left(m_{\lambda}\right)_{\lambda \in N}$ is an increasing sequence. We consider now the sets

$$
\Sigma_{n}=\bigcup_{\lambda=n}^{\infty} \Sigma^{(\lambda)}, \quad n \in N \quad \text { and } \quad \Sigma_{0}=\bigcap_{n \in N} \Sigma_{n} .
$$

We have,

$$
\text { meas } \Sigma_{n} \leqq \sum_{\lambda=n}^{\infty} \operatorname{meas}\left(\Sigma^{(\lambda)}\right) \leqq \sum_{\lambda=n}^{\infty} 1 / 2^{\lambda}=1 / 2^{n-1} 1, \quad \text { meas } \Sigma_{0}=0
$$

(c) Let us now take any point $t_{0} \in\left[\begin{array}{ll}t_{1} & t_{2}\end{array}\right]-\left(\Sigma_{0} \cup T_{0} \cup T_{1} \cup H\right)$. Then there is a real $\sigma>0$ and an integer $n_{0}$ such that $t_{1}<t_{0}-\sigma<t_{0}<t_{0}+\sigma<t_{2}$ and $t_{0} \notin \Sigma^{(\lambda)}$ for every $\lambda \geqq n_{0}$.

For every given $\varepsilon>0$ we take $\lambda \in N$ sufficiently large that $1 / 2^{\lambda}<\varepsilon / 2$ and $t_{0} \notin \Sigma^{(\lambda)}$. Consequently $t_{0} \in\left(I^{(\lambda)}\right)^{0}$ with $I^{(\lambda)} \notin S_{k_{s}^{\lambda}}^{(\lambda)}, s \in N$; hence

$$
\omega\left(x_{k_{s}^{\lambda}}, I^{(\lambda)}\right)<1 / 2^{\lambda}<\varepsilon / 2 \quad \text { for every } s \in N .
$$


Since $\lambda$ is fixed now, for simplicity we shall write $\left(k_{s}^{\lambda}\right)_{s \in N}=\left(k_{s}\right)_{s \in N}$. For every $0<h<\sigma$ we consider the averages

$$
\begin{gathered}
m_{h}=h^{-1} \int_{0}^{h} x^{\prime}\left(t_{0}+\tau\right) d \tau=h^{-1}\left[x_{a}\left(t_{0}+h\right)-x_{a}\left(t_{0}\right)\right] \\
m_{k_{s} h}=h^{-1} \int_{0}^{h} x_{k_{s}}^{\prime}\left(t_{0}+\tau\right) d \tau=h^{-1}\left[x_{k_{s}}\left(t_{0}+h\right)-x_{k_{s}}\left(t_{0}\right)\right] .
\end{gathered}
$$

Now, for an arbitrary fixed $\eta>0$ and for all $0<h<\sigma$ sufficiently small we have

$$
\left|m_{h}-x_{a}^{\prime}\left(t_{0}\right)\right|<\eta / 2 \text { and } \quad\left|x_{s}\left(t_{0}+h\right)-x_{s}\left(t_{0}\right)\right|<h \eta / 4 .
$$

Thus we fix $0<h<\min (\varepsilon, \sigma)$ in such a way that relation (5) holds and moreover $t_{0}+h \varsubsetneqq T_{0} \cup T_{1}$ and $\left[t_{0}, t_{0}+h\right] \subset I^{(\lambda)}$. From (4), for every $t_{0} \leqq t \leqq$ $t_{0}+h$, we have

$$
\left|x_{k_{s}}(t)-x_{k_{s}}\left(t_{0}\right)\right| \leqq \omega\left(x_{k_{s}}, I^{(\lambda)}\right)<\varepsilon / 2, \quad s \in N
$$

Since $t_{0}, t_{0}+h \notin T_{0}$, we can find an integer $s$ such that we have

$$
\left|x_{k_{s}}\left(t_{0}\right)-x\left(t_{0}\right)\right| \leqq \min \{\varepsilon / 2, \eta h / 8\}
$$

and

$$
\left|x_{k_{s}}\left(t_{0}+h\right)-x\left(t_{0}+h\right)\right|<\eta h / 8 \text {. }
$$

Therefore from (6) and (7), for every $t_{0} \leqq t \leqq t_{0}+h$, we have

$\left|x_{k_{s}}(t)-x\left(t_{0}\right)\right| \leqq\left|x_{k_{s}}(t)-x_{k_{s}}\left(t_{0}\right)\right|+\left|x_{k_{s}}\left(t_{0}\right)-x\left(t_{0}\right)\right|<\varepsilon / 2+\varepsilon / 2=\varepsilon$.

By hypothesis we have

$$
x_{k_{s}}^{\prime}(t) \in Q\left(t, x_{k_{s}}(t)\right) \quad \text { for a.a. } t_{0} \leqq t \leqq t_{0}+h ;
$$

hence, from (8) and because $h<\varepsilon$, we have

$$
x_{k_{s}}^{\prime}(t) \in \mathrm{cl} \operatorname{co} \underset{\substack{\left.t \in\left[t_{0}, t_{0}+\varepsilon\right] \\ x \in B\left(x_{0}\right), \varepsilon\right)}}{\bigcup} Q(t, x)=\mathrm{cl} \operatorname{co} Q\left(t_{0}, x\left(t_{0}\right), \varepsilon\right)
$$

for a.a. $t_{0} \leqq t \leqq t_{0}+h$.

Finally we observe that the average $m_{k_{s} h}$ is also a point of the same closed and convex set ([5], p. 288), i.e.

$$
m_{k_{s} h} \in \mathrm{cl} \operatorname{co} Q\left(t_{0}, x\left(t_{0}\right), \varepsilon\right) \text {. }
$$

Now by virtue of (7) and (5) we derive that

$$
\begin{aligned}
\left|m_{k_{s} h}-m_{h}\right|= & h^{-1}\left|\left(x_{k_{s}}\left(t_{0}+h\right)-x_{k_{s}}\left(t_{0}\right)\right)-\left(x_{a}\left(t_{0}+h\right)-x_{a}\left(t_{0}\right)\right)\right| \\
\leqq & h^{-1}\left|x_{k_{s}}\left(t_{0}+h\right)-x\left(t_{0}+h\right)\right|+h^{-1}\left|x_{k_{s}}\left(t_{0}\right)-x\left(t_{0}\right)\right| \\
& +h^{-1}\left|x_{s}\left(t_{0}+h\right)-x_{s}\left(t_{0}\right)\right| \\
\leqq & h^{-1} \eta h / 8+h^{-1} \eta h / 8+h^{-1} \eta h / 4=\eta / 2 .
\end{aligned}
$$


Thus, by (5) and (11), it follows that,

$$
\left|x_{a}^{\prime}\left(t_{0}\right)-m_{k_{s} h}\right| \leqq\left|x_{a}^{\prime}\left(t_{0}\right)-m_{h}\right|+\left|m_{h}-m_{k_{s} h}\right| \leqq \eta / 2+\eta / 2=\eta ;
$$

and, from (10) and (12), that

$$
x_{a}^{\prime}\left(t_{0}\right)=x^{\prime}\left(t_{0}\right) \in\left[\mathrm{clco} Q\left(t_{0}, x\left(t_{0}\right), \varepsilon\right)\right]_{\eta} .
$$

Because $\eta$ is arbitrary, it follows that for every $\varepsilon>0$

$$
x^{\prime}\left(t_{0}\right) \in \mathrm{clco} Q\left(t_{0}, x\left(t_{0}\right), \varepsilon\right) .
$$
derive

Now the function $Q$ satisfies property (Q) at $\left(t_{0}, x\left(t_{0}\right)\right)$; hence from (13) we

$$
x^{\prime}\left(t_{0}\right) \in \bigcap_{\varepsilon>0} \operatorname{clco} Q\left(t_{0}, x\left(t_{0}\right), \varepsilon\right)=Q\left(t_{0}, x\left(t_{0}\right)\right) .
$$

This completes the proof of (2).

Remark 1. In Theorem 1 condition (Q) cannot be replaced by the weaker condition (K) as the following example, from Section 8.8 of [5], shows. We report the example here with some simplifications for the convenience of the reader. Let $n=1$ and $A=[0,1] \times R$, let $C$ be a closed Cantor subset of $[0,1]$ whose measure $|C|$ is positive, and let $C^{\prime}=[0,1]-C$. Then $C^{\prime}$ is the countable union of disjoint subintervals of $[0,1], C^{\prime}=\bigcup_{n \in N} I_{n}$. Let $\sigma(t): C^{\prime} \rightarrow R^{+}$be a continuous and integrable function, which tends to $+\infty$ whenever $t$ tends to an end point of any interval $I_{n}$. Moreover let us suppose that $\lim _{n \rightarrow+\infty} \min \sigma / I_{n}=+\infty$.

Let $Q(t)=\{-1\}$ if $t \in C$, and $Q(t)=\{z \in R: z \geqq \stackrel{n \rightarrow+\infty}{\sigma(t)}\}$ if $t \in C^{\prime}$. Let us extend the function $\sigma$ by taking $\sigma(t)=0$, for $t \in C$, and consider the decomposition of $[0,1]$ into $k$ intervals of equal length: $J_{k}^{s}=\left[t_{k, s-1}, t_{k, s}\right], s=1, \ldots, k$, $t_{k, s}=s / k$. Define $\xi_{k}$ by taking $\xi_{k}(t)=\sigma(t)+v_{k}(t)$, where $v_{k}(t)=-1$ if $t \in C$, and $v_{k}(t)=\left|C \cap J_{k}^{s}\right| /\left|C^{\prime} \cap J_{k}^{s}\right|$ if $t \in C^{\prime} \cap J_{k}^{s}$. Then $\xi_{k}$ is integrable in $[0,1]$, and $\xi_{k}(t) \in Q(t)$ for every $t \in[0,1]$ and $k \in N$.

Let $x_{k}(t)=\int_{0}^{t} \xi_{k}(\tau) d \tau, \quad 0 \leqq t \leqq 1, \quad$ or $x_{k}(t)=x(t)+y_{k}(t)=\int_{0}^{t} \sigma(\tau) d \tau+$ $\int_{0}^{t} v_{k}(\tau) d \tau$. Here $\int_{J_{k}^{s}} v_{k}(t) d t=0 ;$ hence $y_{k}\left(t_{k, s}\right)=0$ for all $s$ and $k$, and $\left|y_{k}(t)\right| \leqq 2 / k$. Hence $x_{k} \rightarrow x$ uniformly on $[0,1]$, as $k \rightarrow+\infty$; moreover all $x_{k}$ and $x$ are AC with $x_{k}^{\prime}(t) \in Q(t), t \in[0,1]$. Now $x^{\prime}(t)=0$ a.e. in $C$, while $Q(t)=\{-1\}$ for $t \in C$. Thus $x^{\prime}(t) \notin Q(t)$ on a subset $C$ of positive measure in $[0,1]$. Note that $\int_{J_{k}^{s}}\left|\nu_{k}(t)\right| d t=2\left|J_{k}^{s} \cap C\right| ;$ hence $V\left(y_{k}\right) \leqq 2$ and $V\left(x_{k}\right) \leqq$ $\int_{0}^{1} \sigma(t) d t+2=V_{0}$, a constant, for all $k$. Here the sets $Q(t, x)$ have property $(\mathrm{K})$ on $[0,1]$; moreover they have property (Q) both on $C$ and on $C^{\prime}$ but not on $[0,1]$. 


\section{An existence theorem of the calculus of variations}

3a. The integral $\mathscr{I}$

Let $\alpha, n$ be integers such that $0 \leqq \alpha \leqq n, n \geqq 1$, and for every $x \in R^{n}$ we write $x=(y, z)$ with $y \in R^{\alpha}, z \in R^{n-\alpha}$. Let $A \subset R^{n+1}$ and $Q: A \rightarrow R^{n}$ be defined as before. Let $M C R^{2 n+1}$ denote the set $M=\{(t, x, \zeta):(t, x) \in A$, $\zeta \in Q(t, x)\}$, and let $F_{0}(t, x, \zeta)$, or $F_{0}: M \rightarrow R$ be a given function.

Let $\Omega$ be a class of admissible functions, i.e. functions $x(t)=(y(t), z(t))$, or $x:\left[t_{1}, t_{2}\right] \rightarrow R^{n}$, such that (i) $y$ is ACg and $z$ is BVC; (ii) $(t, x(t)) \in A, x^{\prime}(t) \in$ $Q(t, x(t))$ a.e. in $\left[t_{1}, t_{2}\right]$; (iii) $F_{0}\left(\cdot, x(\cdot), x^{\prime}(\cdot)\right) \in L_{1}$.

We consider the functional $\mathscr{I}: \Omega \rightarrow \tilde{R}$ defined by

$$
\begin{aligned}
\mathscr{I}(x)=\mathscr{I}(y, z) & =\inf _{\Gamma(x)} \lim _{k \rightarrow \infty} \int_{t_{1}}^{t_{2}} F_{0}\left(t, y_{k}(t), z_{k}(t), y_{k}^{\prime}(t), z_{k}^{\prime}(t)\right) d t \\
& =\inf _{\Gamma(x)} \lim _{k \rightarrow \infty} I\left(y_{k}, z_{k}\right),
\end{aligned}
$$

where $\Gamma(x)$ denotes the class of all sequences $\left(x_{k}\right)_{k \in N}$ such that (a) $x_{k}=\left(y_{k}, z_{k}\right) \in$ $\mathrm{ACg} \cap \Omega, k \in N$; (b) $y_{k} \rightarrow y$ uniformly and $z_{k} \rightarrow z$ pointwise a.e. in $\left[t_{1}, t_{2}\right]$, and where, as stated in (i), $y$ is ACg and $z$ is BVC. If $\Gamma(x)=\emptyset$ we put $\mathscr{I}(x)=+\infty$. We may think of $F_{0}$ as extended to all of $R^{2 n+1}$ by taking $F_{0}=+\infty$ on $R^{2 n+1}-M$. We denote by $\tilde{R}$ the reals with the addition of $+\infty$. Note that if $x \in \operatorname{ACg} \cap \Omega$, then $\Gamma(x) \neq \emptyset$. Moreover, if $A$ is convex, $Q(t, x)=R^{n},(t, x) \in A$, and the integral means $\left(x_{h}\right)_{h>0}$ belong to $\Omega$, then $\Gamma(x) \neq \emptyset$.

The class $\Omega$ is said to be closed if $\Omega$ has the following property (c): if $x_{k}(t)=$ $\left(y_{k}, z_{k}\right)$ is any sequence of (admissible) pairs in $\Omega$ satisfying (a) and (b) above, and if $x=(y, z)$ is admissible, then $x$ belongs to $\Omega$.

The functional $\mathscr{I}$ is modeled on Lebesgue area theory for nonparametric discontinuous surfaces (see CESARI [4]) and it is also close to the concept of integral in the sense of SERRIN [14] when the present mixed convergence is used, uniform on $y$ and pointwise a.e. on $z$.

Note that the generalized weighted variation and length for a BVC curve are particular cases of the functional $\mathscr{I}$. In fact, let $F_{0}(t, v):\left[t_{1}, t_{2}\right] \times R^{n} \rightarrow R_{0}^{+}$be defined by $F_{0}(t, v)=|\varphi(t) \cdot v|$, where $\varphi(t):\left[t_{1}, t_{2}\right] \rightarrow R^{n}$ is continuous. Then (see [1], [13])

$$
\mathscr{I}(z)=\inf _{\Gamma(x)} \lim _{k \rightarrow \infty} \int_{t_{1}}^{t_{2}}\left|z_{k}^{\prime}(t) \cdot \varphi(t)\right| d t=\inf _{\Gamma(x)} \lim _{k \rightarrow \infty} V_{\varphi}^{*}\left(z_{k}\right) \geqq V_{\varphi}^{*}(z),
$$

and moreover

$$
\mathscr{I}(z) \leqq \lim _{h \rightarrow 0} \int_{t_{1}}^{t_{2}}\left|z_{h}^{\prime}(t) \cdot \varphi(t)\right| d t=V_{\varphi}^{*}(z),
$$

where $z_{h}(t)=\frac{1}{h} \int_{0}^{h} z(t+\tau) d \tau$ is the integral mean of $z$. Analogous considerations hold for the length. 
Remark 2. In order to deal with the minimization of the functionals under consideration when initial and terminal values for $x$ are involved, we carry over the definition of the functional $\mathscr{I}$ to the following setting.

Let $\mathscr{N}$ denote a family of subsets $N \subset\left[t_{1}, t_{2}\right]$, with $|N|=0$, which is closed under countable unions. We shall write briefly $\mathscr{N}$-a.e. when we refer to a neglected null set $N \in \mathscr{N}$. Moreover we shall denote by $\mathscr{N}$-AC and $\mathscr{N}$-BVC the family of all the functions which are $\mathscr{N}$-a.e. equal to an $\mathrm{AC}$ function or a $\mathrm{BV}$ function, respectively. that

We consider now the class $\Omega_{\mathscr{N}}$ of all the functions $x(t)=(y(t), z(t))$, such

i) $y \in \mathscr{N}$-AC and $z \in \mathscr{N}$-BVC,

ii) $(t, x(t)) \in A, \mathscr{N}$-a.e. and $x^{\prime}(t) \in Q(t, x(t))$, a.e. in $\left[t_{1}, t_{2}\right]$,

iii) $F_{0}\left(\cdot, x(\cdot), x^{\prime}(\cdot)\right) \in L_{1}$.

For every $x \in \Omega_{\mathscr{N}}$, we shall denote by $\Gamma_{\mathscr{N}}(x)$ the class of all the sequences $\left(x_{k}\right)_{k \in N}$ such that

(a) $x_{k}=\left(y_{k}, z_{k}\right) \in \mathscr{N}-\mathrm{AC}, k \in N$,

(b) $y_{k} \rightarrow y$ uniformly and $z_{k} \rightarrow z$ pointwise $\mathscr{N}$-a.e.

Thus we shall take $\mathscr{I}_{\mathscr{N}}: \Omega_{\mathscr{N}} \rightarrow \tilde{R}$ with

$$
\mathscr{I}_{\mathscr{N}}=\inf _{\Gamma_{\mathscr{N}}(x)} \lim _{k \rightarrow \infty} I\left(x_{k}\right) \quad \text { if } \Gamma_{\mathscr{N}}(x) \neq \emptyset ; \quad \mathscr{I}_{\mathscr{N}}=+\infty \text { otherwise }
$$

Note that, if $\mathcal{N}$ is the family of all the null sets in $\left[t_{1}, t_{2}\right]$, then $\mathscr{I}_{\mathscr{N}}$ is exactly the functional $\mathscr{I}$. Furthermore, if $\mathscr{N}_{1} \subset \mathscr{N}_{2}$, then $\Omega_{\mathscr{N}_{1}} \subset \Omega_{\mathscr{N}_{2}}, \Gamma_{\mathscr{N}_{1}}(x) \subset \Gamma_{\mathscr{N}_{2}}(x)$ and $\mathscr{I}_{\mathscr{N}_{1}}(x) \geqq \mathscr{I}_{\mathscr{N}_{2}}(x)$, for every $x \in \Omega_{\mathscr{N}_{1}}$. Note that $>$ sign may hold, as the Example 2 in Section 3 shows. In this way we get a "spectrum" of integral functionals whose lower and upper lines are $\mathscr{I}$ and $\mathscr{I}_{\{\emptyset}$, respectively. Observe that, if $F_{0}(v)=|v|$, then $\left\{\mathscr{I}_{\mathscr{N}}\right\}_{\mathscr{N}}$ is the "spectrum of variations" whose lower and upper lines are the generalized variation and the classic one, respectively.

In the following, for simplicity, we shall deal with the functional $\mathscr{I}$, but all our results hold for any other functional $\mathscr{I}_{\mathscr{N}}$, as well. In fact, we shall make systematic use of Helly's theorem which guarantees convergence at all points $t \in$ $\left[t_{1}, t_{2}\right]$.

Note that, in this way, we treat also minimization which involves given initial and terminal data for $x$, say $x\left(t_{1}\right) \in B_{1}, x\left(t_{2}\right) \in B_{2}$, with $B_{1}, B_{2}$ closed sets in $R^{n}$. This is the case when the family $\mathscr{N}$ is such that $\bigcup_{N \in \mathscr{N}} N=\left(t_{1}, t_{2}\right)$. Thus, in the computation of the variation $V^{*}(x)$ of $x$ in $\left[t_{1}, t_{2}\right]$ we always take note of the values of $x$ at $t_{1}$ and $t_{2}$. Concerning the convergence of the trace operator, see the known results mentioned in [6a].

Remark 3. We shall see now that, for every $x=(y, z) \in \Omega$, with $\Gamma(x) \neq \emptyset$, there is a sequence $\left(x_{k}\right)_{k \in N} \in \Gamma(x)$ such that

$$
\lim _{k \rightarrow \infty} I\left(x_{k}\right)=\mathscr{I}(x) .
$$

If $\mathscr{I}(x) \in R$, the proof is analogous to that for the case in which $\mathscr{I}(x)=+\infty$. Observe that, by definition, for every $m \in N$ there is a sequence $\left(y_{n}^{m}, z_{n}^{m}\right)_{n \in N} \in$ 
$\Gamma(x)$ such that

$$
\left|\lim _{n \rightarrow \infty} I\left(y_{n}^{m}, z_{n}^{m}\right)-\mathscr{I}(x)\right|<1 / m ;
$$

moreover, by virtue of Severini-Egoroff theorem, we know that $z_{n}^{m} \underset{n \rightarrow \infty}{\rightarrow} z$ almost uniformly, $m \in N$. Thus we can find a set $T_{m} C\left[t_{1}, t_{2}\right]$ and an integer $n_{m}$ such that meas $\left(T_{m}\right)>\left(t_{2}-t_{1}\right)-1 / 2^{m}$ and, for every $n \geqq n_{m}$, we have $\left|I\left(y_{n}^{m}, z_{n}^{m}\right)-\mathscr{I}(x)\right|<2 / m,\left|y_{n}^{m}(t)-y(t)\right|<1 / m, t \in\left[t_{1}, t_{2}\right]$ and $\left|z_{n}^{m}(t)-z(t)\right|$ $<1 / m, t \in T_{m}$.

Let us denote by $\left(x_{k}\right)_{k \in N}$ the sequence $x_{k}=\left(y_{k}, z_{k}\right)=\left(y_{n_{k}}^{k}, z_{n_{k}}^{k}\right), k \in N$. We shall see that $\left(x_{k}\right)_{k \in N}$ is the sequence we were looking for. To do so, we put $T^{k}=$ $\bigcap_{m \geqq k} T_{m}$ and $T=\bigcup_{k \in N} T^{k}$, then meas $\left(T^{k}\right)>\left(t_{2}-t_{1}\right)-1 / 2^{k-1}$ and meas $(T)$ $m \geqq k$
$=t_{2}-t_{1}$. Thus, for every fixed $\varepsilon>0$ and $t \in T$, there is an integer $\bar{k}>1 / \varepsilon$ such that $t \in T_{k}$ for every $k \geqq \bar{k}$ and therefore $\left|z_{k}(t)-z(t)\right|<1 / k<\varepsilon$. In other words $z_{k} \rightarrow z$ pointwise on $T$. Obviously $y_{k} \rightarrow y$ uniformly in $\left[t_{1}, t_{2}\right]$ and hence $\left(x_{k}\right)_{k \in N} \in \Gamma(x)$. Finally, having fixed $\varepsilon>0$ and having taken $k_{\varepsilon} \in N$ such that $k_{\varepsilon}>2 / \varepsilon$, then for every $k \geqq k_{\varepsilon}$, we have $\left|I\left(x_{k}\right)-\mathscr{I}(x)\right|<2 / k_{\varepsilon}<\varepsilon$. The proof is complete.

\section{3b. A lower semicontinuity property of $I$ and $\mathscr{I}$}

As is well known ([5]), closure theorems can be reworded into lower closure theorems and into lower semicontinuity theorems. From the closure Theorem 1 of Section 2 we derive here a lower semicontinuity theorem for the integral $I$ and the relevant inequality $I(x) \leqq \mathscr{I}(x)$, under the assumption $V^{*}\left(x_{k}\right) \leqq V_{0}$, $k \in N$, and the topology under consideration, namely uniform convergence on the components $y^{i}$ and pointwise convergence almost everywhere on the components $z^{j}$.

For the lower semicontinuity theorem we shall need the auxiliary sets

$$
\tilde{Q}(t, x)=\left\{\left(z^{0}, \xi\right) \in R^{n+1}: z^{0} \geqq F_{0}(t, x, \xi), \xi \in Q(t, x)\right\}, \quad(t, x) \in A,
$$

or "augmented" set-valued function $\tilde{Q}: A \rightarrow R^{n+1}$.

Theorem 1 ( $A$ lower semicontinuity theorem). Let $1 \leqq \alpha \leqq n-1$, and assume that: (i) $A$ is closed; (ii) the sets $\tilde{Q}(t, x)$ are closed, convex and have property (Q) with respect to $(t, x)$ at every point $(\bar{t}, \bar{x}) \in A$, with the exception perhaps of a set of points whose t-coordinate lies in a set $H$ of measure zero; (iii) $F_{0}(t, y, z, u, v)$ is lower semicontinuous in $M$ and $\lambda \in L_{1}$ exists such that $F_{0}(t, y, z, u, v) \geqq \lambda(t)$ for all $(t, y, z, u, v) \in M$; (iv) a sequence of vector functions is given

$$
x(t)=(y, z), x_{k}(t)=\left(y_{k}, z_{k}\right), t \in\left[t_{1}, t_{2}\right], y, y_{k}, z_{k} \in \mathrm{ACg}, z \in \mathrm{BVC},
$$

$y_{k} \rightarrow y$ uniformly, $z_{k} \rightarrow z$ pointwise a.e. in $\left[t_{1}, t_{2}\right]$, such that $\left(t, x_{k}(t)\right) \in A$, $x_{k}^{\prime}(t) \in Q\left(t, x_{k}(t)\right)$ a.e. in $\left[t_{1}, t_{2}\right] ;(v) V^{*}\left(x_{k}\right) \leqq V_{0}$ for all $k$ and some constant $V_{0}$. Then $(t, x(t)) \in A, x^{\prime}(t) \in Q(t, x(t))$, a.e. in $\left[t_{1}, t_{2}\right]$ and $\lim _{k \rightarrow+\infty} I\left(x_{k}\right) \geqq I(x)$. Thus, 
if $\left(x_{k}\right)_{k \in N}$ is a sequence as in the definition of $\mathscr{I}(x)$, i.e. $x_{k} \in \operatorname{ACg} \cap \Omega$, and $V^{*}\left(x_{k}\right) \leq$ $V_{0}, k \in N$, then

$$
\lim _{k \rightarrow \infty} I\left(x_{k}\right) \geqq \mathscr{I}(x) \geqq I(x) .
$$

For $\alpha=0$ this statement concerns sequences $z_{k}(t)=\left(z_{k}^{1}, \ldots, z_{k}^{n}\right), \quad z(t)=$ $\left(z^{1}, \ldots, z^{n}\right), \quad t \in\left[t_{1}, t_{2}\right], z_{k} \in \mathrm{ACg}, \quad z \in \mathrm{BVC}$, and the conclusions are still valid.

For $\alpha=n$, this statement concerns sequences $y_{k}(t)=\left(y_{k}^{1}, \ldots, y_{k}^{n}\right), \quad y(t)=$ $\left(y^{1}, \ldots, y^{n}\right), t \in\left[t_{1}, t_{2}\right], y, y_{k} \in \mathrm{ACg}$, and the problem reduces to those discussed in Theorem 10.8.i of [5].

Remark 4. As for Theorem 1, condition (Q) cannot be replaced by the weaker condition (K), as the following example shows. This example is only a modification of the one in Remark 1. Indeed, we take $n=1, F_{0}=0$ and $\tilde{Q}(t)=$ $\left\{\left(z^{0}, z\right): z^{0} \geqq 0, z \in Q(t)\right\}$, where the sets $Q(t)$ are defined in Remark 1. Then, for $x, x_{k}$ as in Remark 1, we have $x_{k}^{\prime}(t) \in Q(t)$, but $x^{\prime}(t) \notin Q(t)$, for $t \in C$, a set of positive measure.

Proof of Theorem 1'. Without loss of generality we can suppose that $i=$ $\lim _{k \rightarrow+\infty} I\left(x_{k}\right)=\lim _{k \rightarrow+\infty} I\left(x_{k}\right)<+\infty$, where $i$ is finite because of (iii). Take $F_{k}(t)=$ $F_{0}\left(t, y_{k}(t), z_{k}(t), y_{k}^{\prime}(t), z_{k}^{\prime}(t)\right), t \in\left[t_{1}, t_{2}\right]$, and note that for $F_{k}(t)=F_{k}^{+}(t)-F_{k}^{-}(t)$, $\left|F_{k}(t)\right|=F_{k}^{+}+F_{k}^{-}, \quad F_{k}^{+}, F_{k}^{-} \geqq 0$, we have $0 \leqq F_{k}^{-}(t) \leqq|\lambda(t)|$; hence $0 \leqq$ $\int_{t_{1}}^{t_{2}} F_{k}^{-}(t) d t \leqq \int_{t_{1}}^{t_{2}}|\lambda(t)| d t$. Since $i$ is finite, we have that $I\left(x_{k}\right) \leqq W_{0}$ for all $k$ and some constant $W_{0}$. Finally,

$$
\begin{aligned}
\int_{t_{1}}^{t_{2}}\left|F_{k}(t)\right| d t & =\int_{t_{1}}^{t_{2}}\left(F_{k}^{+}+F_{k}^{-}\right)(t) d t=\int_{t_{1}}^{t_{2}} F_{k}(t) d t+2 \int_{t_{1}}^{t_{2}} F_{k}^{-}(t) d t \\
& \leqq W_{0}+2 \int_{t_{1}}^{t_{2}}|\lambda(t)| d t<+\infty .
\end{aligned}
$$

Let us consider the AC functions $z_{k}^{0}:\left[t_{1}, t_{2}\right] \rightarrow R$ defined by $z_{k}^{0}(t)=$ $\int_{t_{1}}^{t} F_{0}\left(\tau, x_{k}(\tau), x_{k}^{\prime}(\tau)\right) d \tau, k \in N$. Then $z_{k}^{0}\left(t_{1}\right)=0$ and

$$
V\left(z_{k}^{0}\right)=\int_{i_{1}}^{t_{2}}\left|F_{k}(t)\right| d t \leqq W_{0}+2 \int_{i_{1}}^{t_{2}}|\lambda(t)| d t=V,
$$

$V$ a constant. By Helly's theorem there is a subsequence, say still $(k)$, such that $z_{k}^{0}(t) \rightarrow z^{0}(t)$ pointwise everywhere in $\left[t_{1}, t_{2}\right]$, with $z^{0} \in \mathrm{BV}, z^{0}$ not necessarily continuous, and $z^{0}\left(t_{1}\right)=0$. Note that the functions $\left(z_{k}^{0}, x_{k}\right)_{k \in N}$ are $\mathrm{ACg}$ solutions of the orientor field

$$
\left(t, x_{k}(t)\right) \in A,\left(z_{k}^{0^{\circ}}(t), x_{k}^{\prime}(t)\right) \in \tilde{Q}\left(t, x_{k}(t)\right), \text { a.e. in }\left[t_{1}, t_{2}\right],
$$

where $\tilde{Q}: A \rightarrow R^{n+1}$ is the set-valued function defined by (1). As an application of Theorem 1 we now prove that the limit function $\left(z^{0}, y, z\right)=\left(z^{0}, x\right)$ : 
$\left[t_{1}, t_{2}\right] \rightarrow R^{n+1}$ is again a solution of the orientor field (2), i.e.

or

$$
(t, x(t)) \in A, \quad\left(z^{\prime \prime}(t), x^{\prime}(t)\right) \in \tilde{Q}(t, x(t)) \text { a.e. in }\left[t_{1}, t_{2}\right],
$$

$$
(t, x(t)) \in A, \quad x^{\prime}(t) \in Q(t, x(t)), \quad z^{0 \prime}(t) \geqq F_{0}\left(t, x(t), x^{\prime}(t)\right) \text { a.e. in }\left[t_{1}, t_{2}\right] .
$$

Note that $z^{0 \prime}(t) \geqq F_{0}(t) \geqq \lambda(t)$; hence $F_{0}\left(t, x(t), x^{\prime}(t)\right)$ is summable in $\left[t_{1}, t_{2}\right]$ because it lies between summable functions, and $x:\left[t_{1}, t_{2}\right] \rightarrow R^{n}$ is admissible. Finally, if we take

$$
Z^{0}(t)=z^{0}(t)-\int_{t_{1}}^{t} \lambda(\tau) d \tau, \quad t \in\left[t_{1}, t_{2}\right],
$$

we see that $Z^{0}(t)$ is monotone non-decreasing in $\left[t_{1}, t_{2}\right]$; hence

$$
\begin{aligned}
Z^{0}\left(t_{2}\right) & =Z^{0}\left(t_{2}\right)-Z^{0}\left(t_{1}\right)=z^{0}\left(t_{2}\right)-\int_{t_{1}}^{t_{2}} \lambda(t) d t \\
& =V\left(Z^{0}\right) \geqq \int_{t_{1}}^{t_{2}}\left|z^{0 \prime}(t)-\lambda(t)\right| d t=\int_{t_{1}}^{t_{2}}\left(z^{0 \prime}(t)-\lambda(t)\right) d t \\
& \geqq \int_{t_{1}}^{t_{2}}\left(F_{0}(t)-\lambda(t)\right) d t=\int_{t_{1}}^{t_{2}} F_{0}(t) d t-\int_{t_{1}}^{t_{2}} \lambda(t) d t .
\end{aligned}
$$

Hence $z^{0}\left(t_{2}\right) \geqq \int_{t_{1}}^{t_{2}} F_{0}(t) d t$, and finally

$$
I(x)=\int_{t_{1}}^{t_{2}} F_{0}(t) d t \leqq z^{0}\left(t_{2}\right)=\lim _{k \rightarrow+\infty} z_{k}^{0}\left(t_{2}\right)=\lim _{k \rightarrow+\infty} I\left(x_{k}\right) .
$$

Theorem $1^{\prime}$ is thereby proved.

Theorem $1^{\prime}$ has an important consequence concerning the concept of integral $\mathscr{I}(x)$ defined at the beginning of Section 3. Indeed, as long as we define $\mathscr{F}(x)$ by means of sequences $x_{k}=\left(y_{k}, z_{k}\right) \in \mathrm{ACg}, k \in N$, with equibounded variation, then we can well say that $\mathscr{I}$ is an extension to BVC of the functional $I$; in other words, if $x=(y, z), y, z$ both $\mathrm{ACg}$, then $\mathscr{I}(x)=I(x)$. In fact, from Theorem $1^{\prime}$ we have ${ }^{1} I(x) \leqq \mathscr{F}(x)$, but $\Gamma(x)$ contains now the sequence of repetitions $x_{k}=$ $(y, z), k \in N$; hence $\mathscr{I}(x) \leqq I(x)$, and finally $I(x)=\mathscr{F}(x)$.

\section{3c. The existence theorem}

We now state and prove an existence theorem of the calculus of variations for the integral $\mathscr{I}$. In other words we have to prove that $\mathscr{I}$ has an absolute minimum in $\Omega$. That is we have to prove, under the assumptions that

(a) the infimum $i$ of $I(x)$ in $\operatorname{ACg} \cap \Omega$ is finite; hence there are minimizing sequences $x_{k}=\left(y_{k}, z_{k}\right), k \in N$, in $\Omega$, both $y_{k}, z_{k} \in \mathrm{ACg}$, such that $I\left(x_{k}\right) \rightarrow i$;

(b) for some subsequence, say (k) again, and elements $x=(y, z) \in \Omega, y \in \mathrm{ACg}$, $z \in \mathrm{BVC}$, we have $y_{k} \rightarrow y$ uniformly, $z_{k} \rightarrow z$ pointwise a.e. in $\left[t_{1}, t_{2}\right]$.

${ }^{1}$ This is not true, in general, if assumption (v) of Theorem $1^{\prime}$ is dropped, as Example 3 below shows. 
Since we shall assume that there are such sequences $x_{k}=\left(y_{k}, z_{k}\right)$ with equibounded variations $V\left(x_{k}\right)$, then by Theorem $1^{\prime}$ we know that $I(x) \leqq \mathscr{I}(x)=i{ }^{2}$

To state and prove our existence theorem, we denote by $\left(\gamma_{1}\right),\left(\gamma_{2}\right),\left(\gamma_{3}\right)$ the following alternative assumptions on the function $F_{0}$.

$\left(\gamma_{1}\right)$ There is a scalar function $\phi(\zeta), 0 \leqq \zeta<+\infty$, or $\phi: R_{0}^{+} \rightarrow R$, bounded below, with $\phi(\zeta) / \zeta \rightarrow+\infty$ as $\zeta \rightarrow+\infty$, such that $F_{0}(t, y, z, u, v) \geqq$ $\phi(|u|)$ for all $(t, y, z, u, v) \in M$.

$\left(\gamma_{2}\right)$ For any $\varepsilon>0$ there is an integrable scalar function $\psi_{\varepsilon}(t) \geqq 0$, or $\psi_{\varepsilon}:\left[t_{1}, t_{2}\right] \rightarrow R_{0}^{+}$, such that $|u| \leqq \psi_{\varepsilon}(t)+\varepsilon F_{0}(t, y, z, u, v)$ for all $(t, y, z, u, v) \in M$.

$\left(\gamma_{3}\right)$ For every $\alpha$-vector $p \in R^{\alpha}$ there is an integrable scalar function $\phi_{p}(t) \geqq 0$, or $\phi_{p}:\left[t_{1}, t_{2}\right] \rightarrow R_{0}^{+}$, such that $F_{0}(t, y, z, u, v) \geqq\langle p, u\rangle-\phi_{p}(t)$ for all $(t, y, z, u, v) \in M$.

Note that under condition $\left(\gamma_{1}\right)$ certainly $\phi(\zeta) \geqq \lambda$ for some real constant $\lambda$, and then $F_{0}(t, y, z, u, v) \geqq \phi(|u|) \geqq \lambda$ for all $(t, y, z, u, v)$. Under condition $\left(\gamma_{2}\right)$ and $\varepsilon=1$ we have $|u| \leqq \psi_{1}(t)+F_{0}(t, y, z, u, v)$; hence $F_{0}(t, y, z, u, v) \geqq$ $-\psi_{1}(t)$, a summable function in $\left[t_{1}, t_{2}\right]$. Under condition $\left(\gamma_{3}\right)$ and $p=0$, we have $F_{0}(t, y, z, u, v) \geqq-\phi_{0}(t)$, a summable function in $\left[t_{1}, t_{2}\right]$.

Theorem 2 (An existence theorem). Let $1 \leqq \alpha \leqq n-1$, and assume that (i) $A$ is compact and $M$ is closed; (ii) the sets $\tilde{Q}(t, x)$ are closed and convex and have property $(Q)$ with respect to $(t, x)$ at every point $(t, x)$ of $A$ (with the exception perhaps of a set of points whose t-coordinate lies on a set of measure zero on the $t$-axis); (iii) $F_{0}(t, y, z, u, v)$ is lower semicontinuous in $M$; (iv) $F_{0}$ satisfies one of the growth conditions $\left(\gamma_{1}\right)$, or $\left(\gamma_{2}\right)$, or $\left(\gamma_{3}\right)$. Also we assume that the class $\Omega$ is nonempty and closed, and $(\mathrm{v})$ there exists a constant $W_{0}$ such that for every element $x=(y, z) \in$ $\operatorname{ACg} \cap \Omega$, then $V^{*}(z) \leqq W_{0}$.

Then the functional $\mathscr{I}$ has an absolute minimum $x=(y, z)$ in $\Omega, y \in \mathrm{ACg}$, $z \in \mathrm{BVC}$, and $I(x) \leq \mathscr{J}(x)=i$.

For $\alpha=0$, then $x=z$, requirements $\left(\gamma_{1}\right)$, or alternatively $\left(\gamma_{2}\right),\left(\gamma_{3}\right)$ do not apply, yet the conclusion is still valid if we know that (iv)' there is a summable scalar function $\lambda:\left[t_{1}, t_{2}\right] \rightarrow R$ such that $F_{0}(t, z, \zeta) \geqq \lambda(t)$ for all $(t, z, \zeta) \in M$.

For $\alpha=n$, then $x=y, \Omega$ is a nonempty and closed class of $\mathrm{ACg}$ functions $y(t)=\left(y^{1}, \ldots, y^{n}\right), t \in\left[t_{1}, t_{2}\right]$, condition (v) does not apply, and the problem reduces essentially to those discussed in Theorems 11.1.i and ii of [5].

Note that the condition in (ii) concerning property $(Q)$ for the sets $\tilde{Q}(t, x)$, not only implies that the same sets $\tilde{Q}(t, x)$ are closed and convex, but also that their projections, the sets $Q(t, x)$, also are convex, and that $F_{0}(t, y, z, u, v)$ is convex in $(u, v)$.

\footnotetext{
${ }^{2}$ See Note 1.
} 
Note that for $0 \leqq \alpha \leqq n-1$, if $\left(\mathrm{v}^{\prime}\right)$ there are scalar functions $\psi_{i} \in L_{1}\left(\left[t_{1}, t_{2}\right]\right)$, $i=\alpha+1, \ldots, n$, such that $(t, y, z, u, v) \in M$ implies $v^{i} \geqq \psi_{i}(t)$ a.e. in $\left[t_{1}, t_{2}\right]$, then (v) certainly holds. (Cf. part (g) of proof of Theorem 2 below).

Note that for $1 \leqq \alpha \leqq n-1$, the sets $\tilde{Q}(t, x)$ closed and convex, and $\left(\gamma_{1}\right)$ holds, if $\left(\mathrm{v}^{\prime \prime}\right)$ there are constants $L_{i}$ such that, a.e. in $\left[t_{1}, t_{2}\right],(t, y, z, u, v) \in M$ implies $v^{i} \geqq L_{i}, \quad i=\alpha+1, \ldots, n$, then both (ii) and (v) hold. (Cf. part (h) of proof of Theorem 2 below).

Remark 5. If $\alpha=n, x=y$, note that Theorems 11.1 , i, ii of [5] are proved under weaker assumptions on the function $F_{0}$ and definitively less information on the sets $\tilde{Q}(t, x)$. In particular in Theorem 11.1.ii, under none of the assumptions $\left(\gamma_{1}\right),\left(\gamma_{2}\right)$, $\left(\gamma_{3}\right)$ is it needed to verify that the sets $\tilde{Q}(t, x)$ have property (Q). Indeed, a different topology is used on the functions $y_{k}$, namely $y_{k} \rightarrow y$ uniformly and $y_{k}^{\prime} \rightarrow y^{\prime}$ weakly in $L_{1}$. Then, in terms of the equivalence theorem ([5], Theorem 10.3.i; see also CESARI \& PUCCI [7]), these sets $\tilde{Q}(t, y)$ are shown to have augmented sets $\tilde{\mathscr{Q}}(t, y)$ which have property $(\mathrm{Q})$ with respect to $y$, a.e. in $t$ (see [5], proof of Theorem 10.7.i.).

Proof of Theorem 2. Without loss of generality we can suppose that there is an element $x \in \Omega$ such that $\Gamma(x) \neq \emptyset$. Let $i=\inf _{\Omega} \mathscr{I}(x)$; then $-\infty \leqq i<+\infty$. Let $\left(\bar{x}_{k}\right)_{k \in N}$ be a minimizing sequence, i.e. $\mathscr{I}\left(\bar{x}_{k}\right) \rightarrow i$ as $k \rightarrow \infty$. We divide the proof into parts.

(a) First note that we can find a sequence $\left(x_{k}\right)_{k \in N}$ in $\operatorname{ACg} \cap \Omega$ such that $I\left(x_{k}\right) \rightarrow i$ as $k \rightarrow \infty$. In order to see this, note that for every $k \in N$, there is an integer $n_{k}$ such that $\left|\mathscr{I}\left(\bar{x}_{n_{k}}\right)-i\right|<1 / k$, and moreover (see Remark 2) there is a sequence $\left(x_{n}^{k}\right)_{n \in N}$ in $\operatorname{ACg} \cap \Omega$ such that $I\left(x_{n}^{k}\right) \rightarrow \mathscr{I}\left(\bar{x}_{n_{k}}\right)$ as $n \rightarrow \infty$. Thus, for every $k \in N$, there is an integer $\bar{n}=\bar{n}(k)$ such that $\left|I\left(x_{n}^{k}\right)-i\right|<2 / k$ (or $I\left(x_{\bar{n}}^{k}\right)<k$ if $\left.i=-\infty\right)$. Let $x_{k}=x_{\bar{n}}^{k}, k \in N$. Then $x_{k} \in \operatorname{ACg} \cap \Omega$, and $\left|I\left(x_{k}\right)-i\right|$ $<2 / k, k \in N$. Without loss of generality we can suppose that $x_{k}$ is $\mathrm{AC}_{3} k \in N$, and, since $A$ is compact, the sequence $\left(x_{k}\right)_{k \in N}, x_{k}=\left(y_{k}, z_{k}\right)$, is equibounded.

(b) By virtue of hypothesis $\left(\gamma_{1}\right)$, we prove, as in Theorem 10.4.i of [5] that the sequence $\left(y_{k}\right)_{k \in N}$ is equi-absolutely continuous. Thus, $V\left(y_{k}\right) \leqq V_{1}$ for all $k \in N$ and some constant $V_{1}$. Moreover, since $\left(y_{k}\right)_{k \in N}$ is equibounded, there is a subsequence, say still $\left(y_{k}\right)_{k \in N}$, such that $y_{k} \rightarrow y$ uniformly in $\left[t_{1}, t_{2}\right]$, with $y \in \mathrm{AC}$.

Moreover, the assumption (v) shows that $V\left(z_{k}\right) \leqq W_{0}$ for all $k \in N$. Hence, by Helly's theorem (see [5], Theorem 15.1.i) there is a subsequence, say still $\left(z_{k}\right)_{k \in N}$, such that $z_{k}(t) \rightarrow z(t)$ pointwise everywhere in $\left[t_{1}, t_{2}\right]$ with $z \in \mathrm{BV}$, not necessarily continuous.

(c) Now the function $\phi$ in $\left(\gamma_{1}\right)$ is bounded below, say $\phi(\zeta) \geqq \lambda, \lambda \in R$; hence $F_{k}(t)=F\left(t, y_{k}(t), z_{k}(t), y_{k}^{\prime}(t), z_{k}^{\prime}(t)\right) \geqq \phi\left(\left|y_{k}^{\prime}(t)\right|\right) \geqq \lambda$ for $k \in N$ and $t \in\left[t_{1}, t_{2}\right]$. Consequently, for $F_{k}(t)=F_{k}^{+}(t)-F_{k}^{-}(t), F_{k}^{-} \geqq 0, F_{k}^{+} \geqq 0,\left|F_{k}(t)\right|=F_{k}^{+}(t)+$ 
$F_{k}^{-}(t)$, we have $F_{k}(t) \geqq \lambda, F_{k}^{-}(t) \leqq|\lambda|$. Since $\int_{t_{1}}^{t_{2}} F_{k}(t) d t \rightarrow i$ as $k \rightarrow \infty$, we derive $i \geqq \lambda\left(t_{2}-t_{1}\right)$, and thus $i$ is finite. Without loss of generality we can assume that $\int_{t_{1}}^{t_{2}} F_{k}(t) d t \leqq i+1$ for all $k \in N$, and then

$$
\begin{gathered}
\int_{t_{1}}^{t_{2}} F_{k}^{+}(t) d t=\int_{t_{1}}^{t_{2}} F_{k}(t) d t-\int_{t_{1}}^{t_{2}} F_{k}^{-}(t) d t \leqq i+1+|\lambda|\left(t_{2}-t_{1}\right), \\
\int_{t_{1}}^{t_{2}}\left|F_{k}(t)\right| d t \leqq i+1+2|\lambda|\left(t_{2}-t_{1}\right) .
\end{gathered}
$$

Let us consider now the AC functions $z_{k}^{0}:\left[t_{1}, t_{2}\right] \rightarrow R$ defined by $z_{k}^{0}(t)=$ $\int_{i_{1}}^{t} F_{0}\left(\tau, x_{k}(\tau), x_{k}^{\prime}(\tau)\right) d \tau, k \in N$. Then $V\left(z_{k}^{0}\right)=\int_{t_{1}}^{t_{2}}\left|F_{k}(t)\right| d t \leqq i+1+2|\lambda|\left(t_{2}-t_{1}\right)$ $=V, k \in N$. Again, by Helly's theorem there is a subsequence, say still $\left(z_{k}^{0}\right)_{k \in N}$, such that $z_{k}^{0}(t) \rightarrow z^{0}(t)$ pointwise everywhere in $\left[t_{1}, t_{2}\right]$, with $z^{0} \in \mathrm{BV}$ (not necessarily continuous).

(d) Note that the functions $\left(z_{k}^{0}, x_{k}\right)_{k \in N}$ are AC solutions of the orientor field $\left(t, x_{k}(t)\right) \in A, \quad\left(z_{k}^{0 \prime}(t), x_{k}^{\prime}(t)\right) \in \tilde{Q}\left(t, x_{k}(t)\right)$, a.e. in $\left[t_{1}, t_{2}\right]$, where $\tilde{Q}: A \rightarrow R^{n+1}$ is the set-valued function defined by $\tilde{Q}(t, x)=\left\{(r, \zeta): \zeta \in Q(t, x), r \geqq F_{0}(t, x, \zeta)\right\}$ $=$ epi $F_{0}(t, x, \cdot)_{/ Q(t, x)}$. By (ii) the sets $\tilde{Q}(t, x)$ have property $(Q)$ with respect to $(t, x)$ in $A$, for a.e. $t$.

(e) As an application of Theorem 1, we see that the limit function $\left(z^{0}, y, z\right)=\left(z^{0}, x\right):\left[t_{1}, t_{2}\right] \rightarrow R^{n+1}$ is again a solution of the orientor field $(t, x(t)) \in A, \quad\left(z^{0 \prime}(t), x^{\prime}(t)\right) \in \tilde{Q}(t, x(t))$, a.e. in $\left[t_{1}, t_{2}\right]$, i.e. $(t, x(t)) \in A, \quad x^{\prime}(t) \in$ $Q(t, x(t)), \quad z^{0^{\prime}}(t) \geqq F\left(t, x(t), x^{\prime}(t)\right), \quad$ a.e. in $\left[t_{1}, t_{2}\right]$. Note that $z^{0^{\prime}}(t) \geqq F_{0} \geqq \lambda$; hence $F_{0}\left(t, x(t), x^{\prime}(t)\right)$ is summable in $\left[t_{1}, t_{2}\right]$ and the function $x:\left[t_{1}, t_{2}\right] \rightarrow R^{n}$ is admissible. Since the class $\Omega$ is closed, we conclude that $x \in \Omega$.

(f) Finally, by definition, $\left(x_{k}\right)_{k \in N} \in \Gamma(x)$ and $i \leqq \mathscr{I}(x) \leqq \lim _{k \rightarrow \infty} I\left(x_{k}\right)=i$, or $\mathscr{I}(x)=i$ and the proof is complete.

(g) Let us prove now that, for $0 \leqq \alpha \leqq n-1, A$ compact and property $\left(\mathrm{v}^{\prime}\right)$, then $V\left(z_{k}\right) \leqq V_{2}, k \in N$, for some constant $V_{2}$. Indeed, since $z_{k}$ is AC, we have (writing $z_{k}$ for any of its components $z_{k}^{i}, i=\alpha+1, \ldots, n$ ),

$$
z_{k}\left(t_{2}\right)-z_{k}\left(t_{1}\right)=\int_{t_{1}}^{t_{2}} z_{k}^{+}(t) d t-\int_{t_{2}}^{t_{2}} z_{k}^{\prime-}(t) d t
$$

and

$$
V\left(z_{k}\right)=\int_{t_{1}}^{t_{2}} z_{k}^{\prime+}(t) d t+\int_{t_{1}}^{t_{2}} z_{k}^{\prime-}(t) d t .
$$


Thus, by condition $\left(\mathrm{v}^{\prime}\right)$, we have

$$
V\left(z_{k}\right)=2 \int_{i_{1}}^{t_{2}} z_{k}^{\prime}(t) d t+z_{k}\left(t_{2}\right)-z_{k}\left(t_{1}\right) \leqq 2 \int_{t_{1}}^{t_{2}} \psi^{-}(t) d t+\operatorname{diam} A .
$$

(h) Let us prove that, if $1 \leqq \alpha \leqq n-1, A$ compact, the sets $\tilde{Q}(t, x)$ closed and convex, and $\left(\gamma_{1}\right)$ and $\left(\mathrm{v}^{\prime \prime}\right)$, then not only (v) but also (ii) holds, that is, the sets $\tilde{Q}(t, x)$ have property (Q) with respect to $(t, x)$. Indeed, we have $F_{0}(t, x, u, v) \geqq$ $\phi(|u|), v^{i} \geqq L_{i}, \quad i=\alpha+1, \ldots, n$, for all $(t, x, u, v) \in M$, and by virtue of Theorem 10.5.ii (second version) of [5] (with the variable $x$ replaced by $(t, x)$ ), the thesis follows.

Remark 6 . The hypothesis (v) can be replaced by the weaker assumption $\left(\mathrm{v}^{\prime \prime \prime}\right)$ the level sets $L_{K}=\{x=(y, z) \in \operatorname{ACg} \cap \Omega: I(x) \leqq K\}$ are equibounded in variation. In fact, as can be seen by the proof, we use condition (v) only to guarantee that every minimizing sequence of $\mathrm{ACg}$ curves $x=(y, z)$ is equibounded in variation.

We shall illustrate now three situations in which even condition $\left(v^{\prime \prime \prime}\right)$ can be dropped.

a) Let $F_{0}: R \rightarrow R_{0}^{+}$be a convex function then $F_{0}$ is the least upper bound of its support straight lines, i.e.

$$
F_{0}(v)=\sup \left\{\varphi(v): \varphi(w)=a w+b \leqq F_{0}(w), w \in R\right\} .
$$

Thus, either $F_{0}(v)=$ const, and then Theorem 2 is trivial, or $F_{0}(v) \geqq a v+b$, $v \in R, a \neq 0$. But in this last case, it is easy to see that every minimizing sequence of $\mathrm{AC}$ curves is equibounded in variation.

b) Let consider now an integrand $F_{0}$ which does not depend on the variable $z$ and does satisfy the condition

$$
\left|v_{1}\right| \leqq\left|v_{2}\right| \text { implies } F_{0}\left(t, v_{1}\right) \leqq F_{0}\left(t, v_{2}\right) .
$$

Then condition $\left(\mathrm{v}^{\prime \prime \prime}\right)$ in Theorem 2 can be omitted, provided we suppose that the sets $Q(t, x)$ are such that if $v \in Q(t, x)$ and $|w| \leqq|v|$, then $w \in Q(t, x)$. In fact, in the present case, we can find a minimizing sequence of $\mathrm{AC}$ curves with equibounded variation. In order to see this, given any sequence of $\mathrm{AC}$ curves such that $I\left(z_{k}\right) \rightarrow i$ as $k \rightarrow+\infty$, it is sufficient to alter the sequence $\left(z_{k}\right)_{k \in N}$ in the following way. For simplicity we write $z_{k}$ for any of its components $z_{k}^{i}$, $i=1, \ldots, n$. Let us suppose first that $z_{k}\left(t_{1}\right)<z_{k}\left(t_{2}\right)$, take

and

$$
\bar{t}=\max \left\{t \in\left[t_{1}, t_{2}\right]: z_{k}(t)=z_{k}\left(t_{1}\right)\right\}, \overline{\bar{t}}=\min \left\{t \in\left[\bar{t}, t_{2}\right]: z_{k}(t)=z_{k}\left(t_{2}\right)\right\}
$$

$$
\bar{z}_{k}(t)= \begin{cases}z_{k}\left(t_{1}\right), & t \in\left[t_{1}, \bar{t}\right], \\ \max \left\{z_{k}(\tau),\right. & \tau \in[\bar{t}, t]\}, \quad t \in[\bar{t}, \overline{\bar{t}}], \\ z_{k}\left(t_{2}\right), & t \in\left[\overline{\bar{t}}, t_{2}\right] .\end{cases}
$$


If $z_{k}\left(t_{1}\right)>z_{k}\left(t_{2}\right)$, we define $\bar{z}_{k}$ analogously by substituting min for max in (3). Finally, if $z_{k}\left(t_{1}\right)=z_{k}\left(t_{2}\right)$, we take $\bar{z}_{k}(t)=z_{k}\left(t_{1}\right), t \in\left[t_{1}, t_{2}\right]$. Observe that, in any case, $\bar{z}_{k}$ is again $\mathrm{AC}$ and moreover it is monotone and $\left|\bar{z}_{k}^{\prime}(t)\right| \leqq\left|z_{k}^{\prime}(t)\right|$, a.e. in $\left[t_{1}, t_{2}\right]$. Therefore $V\left(z_{k}\right) \leqq \operatorname{diam} A, k \in N$, and, by virtue of the assumption on the integrand $F$, we have $I\left(\bar{z}_{k}\right) \leqq I\left(z_{k}\right), k \in N$. This proves that $\left(\bar{z}_{k}\right)_{k \in N}$ is still a minimizing sequence.

c) Let $F_{0}(t, z, v): M \rightarrow R^{+}$subjected to the growth condition $F_{0}(t, z, v) \geqq$ $a|v|+b(t)$, with $a>0$ and $b \in L_{1}$. In this case condition $\left(\mathrm{v}^{\prime \prime \prime}\right)$ is trivially satisfied.

Remark $6^{\prime}$. Note that we may drop the requirement that $A$ be bounded if we know that there is a minimizing sequence $x_{k}=\left(y_{k}, z_{k}\right), y_{k}, z_{k} \in \mathrm{AC}$, with $I\left(x_{k}\right) \rightarrow i$, which is equibounded. Thus the assumptions " $A$ compact and (v)" can be replaced by the weaker conditions: $A$ closed and

$\left(\mathrm{v}^{\prime}\right)$ the level sets $L_{K}=\{x=(y, z) \in \mathrm{ACg} \cap \Omega: I(x) \leqq K\}$ are bounded in the norm $\|x\|=\left|x_{e}\left(t_{1}\right)\right|+V^{*}(x)$, where $x_{e}\left(t_{1}\right)=$ lim ess $x(t)$.

Remark 7. Note that we consider the infimum $i$ of $I(x)$ in the class $\operatorname{ACg} \cap \Omega$ and we prove in Theorem 2 under the hypotheses that there is some element $x=(y, z)$ in $\Omega, y \in \mathrm{ACg}, z \in \mathrm{BVC}$, and some sequence $x_{k}=\left(y_{k}, z_{k}\right), y_{k}$, $z_{k} \in \mathrm{ACg}, k \in N$, in $\Omega$ with $I\left(x_{k}\right) \rightarrow i$, and $I(x) \leqq \mathscr{I}(x)=i$.

In other words, under the assumptions of Theorem 2 , the infimum $i$ is attained by $\mathscr{I}$, or $\mathscr{H}(x)=i$, while $I(x)$ may have a value equal to or less than $i$. In Examples 4 and 5 below $I(x)=\mathscr{I}(x)=i$. However, it may well happen that $I(x)<\mathscr{I}(x)=i$ as Example 1 below shows. Note that if we denote by $i_{0}$ the infimum of $I(x)$ in the class $\Omega$, then $\operatorname{ACg} \cap \Omega \subset \Omega$; hence $i_{0} \leqq i$. We shall see in Example 2 below that possibly $i_{0}<i$, and that both can be attained, say $I(x)=i_{0}$ and $I(\bar{x}) \leqq \mathscr{I}(\bar{x})=i$, possibly by different $x, \bar{x} \in \Omega$. Also note that for $\bar{x}$ optimal for $\mathscr{I}$ under the assumptions of the present paper, we certainly have $i_{0} \leqq I(\bar{x}) \leqq \mathscr{I}(\bar{x})=i$.

Example 1. Let us show that, if $i$ is the infimum of $I(x)$ in $\operatorname{ACg} \cap \Omega$ (and therefore the infimum of $\mathscr{I}(x)$ in $\Omega$ ), and $x \in \Omega$ is a minimizing element, then it may happen that $I(x)<\mathscr{I}(x)=i$.

Let us consider the problem of minimizing the length of the plane curves $z^{1}=z^{1}(t), z^{2}=z^{2}(t), 0 \leqq t \leqq 1$, joining two given points, say $(0,0)$ and $(1,1)$, or

$$
\begin{gathered}
I(x)=\int_{0}^{1}\left[\left(z^{1^{\prime}}(t)\right)^{2}+\left(z^{2^{\prime}}(t)\right)^{2}\right]^{1 / 2} d t, \\
z^{1}(0)=0, \quad z^{2}(0)=0, \quad z^{1}(1)=1, \quad z^{2}(1)=1 .
\end{gathered}
$$

Here, for $z^{1}, z^{2} \in \mathrm{AC}$, the infimum of $I$ is $i=\sqrt{2}$, and this infimum is attained not only by the obvious solution $z^{1}(t)=z^{2}(t)=t, 0 \leqq t \leqq 1$, but also by the infinitely many solutions $z^{1}(t)=z^{2}(t)=\xi(t), 0 \leqq t \leqq 1, \quad \xi \in \mathrm{AC}$, monotone nondecreasing with $\xi(0)=0, \xi(1)=1$; hence $z^{1}(0)=0, z^{2}(0)=0, z^{1}(1)=1$, 
$z^{2}(1)=1$, and

$$
I(z)=\sqrt{2} \int_{0}^{1}\left|\xi^{\prime}\right| d t=\sqrt{2} \int_{0}^{1} \xi^{\prime} d t=\sqrt{2}=i .
$$

On the other hand, let us consider the usual ternary Cantor function $\varphi(t), 0 \leqq$ $t \leqq 1, \varphi(0)=0, \varphi(1)=1$, continuous, monotone non decreasing, with derivative zero a.e. in $[0,1], \varphi \mathrm{BV}$ and not AC. Let $\xi_{k}(t), 0 \leqq t \leqq 1, k \in N$, be a sequence of monotone nondecreasing $A C$ approximations of $\varphi$ with $\xi_{k}(0)=0$, $\xi_{k}(1)=1$, and $\xi_{k} \rightarrow \varphi$ uniformly in $[0,1]$. Now we take the sequence of $\mathrm{AC}$ functions $z_{k}^{1}(t)=z_{k}^{2}(t)=\xi_{k}(t), 0 \leqq t \leqq 1, k \in N$. For $z_{k}=\left(z_{k}^{1}, z_{k}^{2}\right)=\left(\xi_{k}, \xi_{k}\right)$, $z=\left(z^{1}, z^{2}\right)=(\varphi, \varphi)$, we have

$$
I\left(z_{k}\right)=\sqrt{2} \int_{0}^{1}\left|\xi_{k}^{\prime}(t)\right| d t=\sqrt{2} \int_{0}^{1} \xi_{k}^{\prime}(t) d t=\sqrt{2} ;
$$

$z_{k} \rightarrow z$, i.e. $z_{k}^{1} \rightarrow z^{1}, z_{k}^{2} \rightarrow z^{2}$ uniformly; hence pointwise in $[0,1]$

$$
I\left(z_{k}\right) \rightarrow i=\sqrt{2} \text {. }
$$

Thus, in the terms of the beginning of Section $3, \mathscr{I}(\varphi)=i=\sqrt{2}, I(\varphi)=0$; hence $I(\varphi)<\mathscr{A}(\varphi)=i$.

\section{Example 2. Suppose}

$$
A=[0,1]^{2} \cap\left\{(t, x) \in R^{2}: t-\frac{1}{3} \leqq z \leqq t+\frac{1}{3}\right\}, Q(t, x)=R, n=1,
$$

and let $F_{0}(v): R \rightarrow R$ be defined by $F_{0}(v)=|v|$. In this case (see Remark 2) the functional $\mathscr{I}$ is the generalized variation. Let $\varphi(t):[0,1] \rightarrow[0,1]$ be the usual ternary Cantor function. Then $\varphi$ is continuous, $\mathrm{BV}$ and not $\mathrm{ACg}$, with $\varphi^{\prime}(t)=0$, a.e. in $[0,1]$, and graph $\varphi \subset A$. Thus $I(\varphi)=0$, i.e.; if $i_{0}$ denotes the infimum of $I(x)$ in $\Omega$, then $I(\varphi)=i_{0}=0$. On the other hand, if $i$ denotes the infimum of $I(x)$ in $\mathrm{ACg} \cap \Omega$ then by Theorem 2, $\mathscr{I}$ also attains its infimum at some minimizing element $\bar{z} \in \Omega$, and $\mathscr{F}(\bar{z})=i$. Certainly $i>0$ (hence $i>i_{0}$ ), since $i=0$ would imply $\bar{z}^{\prime}(t)=0$ a.e., $\bar{z}(t)=$ const. a.e., and this is not possible given the shape of the set $A$. It is easy to see that $i=2 / 3$ and that a minimizing element is $\bar{z}(t)=0$ for $0 \leqq t \leqq 1 / 3, \bar{z}(t)=t-1 / 3$ for $1 / 3<t \leqq 1$, with $I(\bar{z})=\mathscr{I}(\bar{z})=2 / 3$. Note that if we consider the same problem with boundary data $z(0)=0,=z(1)=1$, then $\varphi$ satisfies the same data; h ence $I(\varphi)=i_{0}=0$ as before. On the other hand, it is easy to see that the new infimum is now $i=1$, and that a minimizing element is $\bar{z}(t)=0$ for $0 \leqq t \leqq 1 / 3, \bar{z}(t)=t-\frac{1}{3}$ for $1 / 3 t<1, \bar{z}(1-0)=2 / 3, \bar{z}(1)=1$ with a jump of $1 / 3$ at $t=1$, and $\mathscr{I}(\bar{z})=1=i$, $I(\bar{z})=2 / 3$ and again $I(\bar{z})<\mathscr{I}(\bar{z})=1$ as in Example 1 . Thus $0=i_{0}=I(\varphi)<$ $I(\bar{z})<\mathscr{J}(\bar{z})=i=1$.

Example 3. We show here an example in which occurs the "non natural" situation $i=\mathscr{I}(x)<I(x)$. In this example $A$ is compact, but the sets $\tilde{Q}$ do not have the property $(Q)$, and for a minimizing sequence of AC functions $x_{k}=\left(y_{k}, z_{k}\right)$, $k \in N$, the total variations $V^{*}\left(z_{k}\right)$ are not equibounded, and the sequence $z_{k}^{\prime}, k \in N$, is not equibounded below. 
Let $A=[0,2 \pi] \times[-1,1]^{2}, \quad Q(t, x)=R^{2}, \quad(t, x) \in A, \quad$ and let $F_{0}(z, v)$ : $[-1,1]^{2} \times R^{2} \rightarrow R^{+}$be defined by

$$
F_{0}\left(z_{1}, z_{2}, v_{1}, v_{2}\right)=\exp \left(z_{1} v_{2}-z_{2} v_{1}\right) .
$$

We consider now the sequence $\left(z_{k}\right)_{k \in N}$ given by

$$
z_{k}^{1}(t)=r_{k} \sin k t, \quad z_{k}^{2}(t)=r_{k} \cos k t, \quad 0 \leqq t \leqq 2 \pi, k \in N,
$$

where $r_{k}=k^{-1 / 3}$. Note that $z_{k}^{1^{\prime}}(t)=r_{k} k \cos k t, z_{k}^{2^{\prime}}(t)=-r_{k} k \sin k t$, and $z_{k}^{1} z_{k}^{2^{\prime}}-z_{k}^{2} z_{k}^{1^{\prime}}=-r_{k}^{2} k=-k^{1 / 3} \rightarrow-\infty$. Therefore we have

$$
0 \leqq \mathscr{I}\left(z_{k}\right) \leqq I\left(z_{k}\right)=\int_{0}^{2 \pi} F_{0}\left(z_{k}(t), z_{k}^{\prime}(t)\right) d t=2 \pi \exp \left(-k^{1 / 3}\right) \rightarrow 0,
$$

as $K \rightarrow \infty$. Thus $i=0$. Note that $V\left(z_{k}^{1}\right)=V\left(z_{k}^{2}\right)=4 r_{k} k=4 k^{2 / 3} \rightarrow+\infty$ as $k \rightarrow \infty$. Since $r_{k} \rightarrow 0$ as $k \rightarrow \infty$, if we take $z=\left(z^{1}, z^{2}\right)$ with $z^{1}(t)=z^{2}(t)$ $=0, t \in\left[t_{1}, t_{2}\right]$, then $z$ is $\mathrm{AC}$ in $[0,2 \pi]$ and $z_{k} \rightarrow z$ uniformly, hence $\mathscr{I}(z)=0$. But $I(z)=\int_{0}^{2 \pi} \exp (0) d t=2 \pi>0$. Moreover, for every $z \in$ BVC we certainly

$$
I(z)=\int_{0}^{2 \pi} \exp \left(z_{1}(t) z_{2}^{\prime}(t)-z_{2}(t) z_{1}^{\prime}(t)\right) d t>0
$$

We shall give now two examples which illustrate Theorem 2 and show that, in general, the minimum of $\mathscr{I}$ is attained by a BVC function, not necessarily $\mathrm{ACg}$.

Example 4. Let $A=[0,2] \times[0,1], \quad n=1, \quad \alpha=0, \quad \psi(t)=0 . \quad Q(t, x)=$ $[0,+\infty)$ for $(t, x) \in A, M=A \times[0,+\infty)$, with boundary conditions $x(0)=0$, $x(2)=1$. Let $F_{0}(t, v)$ be defined by $F_{0}(t, v)=|1-t||v|$ for $(t, v) \in M$. Thus the functional $\mathscr{I}(x)$ is nonnegative. Note that, for the sequence $z_{k}:[0,2] \rightarrow R$, $k \in N$, defined by $z_{k}(t)=0$ for $t \in[0,1-1 / k] ; z_{k}(t)=1$ for $t \in[1,2]$, $z_{k}(t)=1-k+k t$ for $t \in(1-1 / k, 1)$, we have

$$
0 \leqq \mathscr{I}\left(z_{k}\right)=I\left(z_{k}\right)=\int_{1-1 / k}^{1}(1-t) k d t=1 / 2 k \rightarrow 0 \text { as } k \rightarrow \infty .
$$

Thus the infimum $i$ of $\mathscr{I}$ is zero, and $z_{k}$ is a minimizing sequence. The minimum is attained by the discontinuous function $z:[0,2] \rightarrow R$ defined by $z(t)=0$ for $t \in[0,1), z(t)=1$ for $t \in[1,2]$. In other words $I(z)=\mathscr{I}(z)=0$.

Example 5. Let $A=[-1,1] \times[0,1], \quad n=1, \quad \alpha=0, \quad \psi(t)=0, \quad Q(t, x)=$ $[-1,+\infty), M=A \times[-1,+\infty), \quad F_{0}(t, v)=|t| v^{2}, \quad F_{0} \geqq 0 . \quad$ with boundary conditions $x(-1)=0, x(1)=1$. The functional $\mathscr{I}(z)$ is nonnegative. Note that for the sequence $z_{k}:[-1,1] \rightarrow R, k \in N$, defined by $z_{k}(t)=0$ for $t \in[-1,1 / k]$; $z_{k}(t)=(\log k)^{-1} \log t+1$, for $t \in(1 / k, 1]$, we have

$$
0 \leqq \mathscr{I}\left(z_{k}\right)=I\left(z_{k}\right)=\int_{1 / k}^{1}(\log k)^{-2} / t d t=(\log k)^{-1} \rightarrow 0 \quad \text { as } k \rightarrow \infty .
$$


Thus the infimum $i$ of $\mathscr{I}$ is zero and $\left(z_{k}\right)_{k \in N}$ is a minimizing sequence. The minimum is attained by the discontinuous function $z:[-1,1] \rightarrow R$, defined by $z(t)=$ 0 for $t \in[-1,0], z(t)=1$ for $t \in(0,1]$. In other words, $I(z)=\mathscr{A}(z)=i$. For this example of. [5], Section 1.1, no. 4 .

\section{An existence theorem for problems of optimal control}

As above, let $\alpha, n, 0 \leqq \alpha \leqq n$, be given integers and, for every $x \in R^{n}$, let $x=(y, z)$ with $y \in R^{x}$ and $z \in R^{n-x}$. Let $A$ be a compact subset of the $(t, x)$ space such that its projection onto the $t$-axis contains the fixed interval $\left[t_{1}, t_{2}\right]$. Let $U(t, x),(t, x) \in A, U(t, x) \subset R^{m}$, or $U: A \rightarrow R^{m}$ be a given set-valued function and let $M_{0}$ denote the set

$$
M_{0}=\{(t, x, w):(t, x) \in A, w \in U(t, x)\} \subset R^{1+n+m} .
$$

Let $f_{0}(t, x, w), f(t, x, w)=\left(f_{1}, \ldots, f_{n}\right)$ be given functions defined on $M_{0} \subset R^{1+n+m}$. Let $\Omega_{0}$ be a class of admissible systems $(y(t), z(t), w(t)), t \in\left[t_{1}, t_{2}\right]$, i.e. functions $x(t)=(y(t), z(t))$, or $x:\left[t_{1}, t_{2}\right] \rightarrow R^{n}, w:\left[t_{1}, t_{2}\right] \rightarrow R^{m}$, such that (i) $y \in \mathrm{ACg}$, $z \in \mathrm{BVC}, w$ is measurable; (ii) $(t, y(t), z(t)) \in A, w(t) \in U(t, y(t), z(t))$, a.e. in $\left[t_{1}, t_{2}\right]$; (iii) $x^{\prime}(t)=f(t, x(t), w(t))$, a.e. in $\left[t_{1}, t_{2}\right], f_{0}(\cdot, x(\cdot), w(\cdot)) \in L_{1}\left(\left[t_{1}, t_{2}\right]\right)$.

We consider the functional $\mathscr{I}_{0}: \Omega_{0} \rightarrow R$ defined by

$$
\begin{aligned}
\mathscr{I}_{0}(x) & =\mathscr{I}_{0}(y, z)=\inf _{\Gamma_{0}(x)} \lim _{k \rightarrow \infty} \int_{t_{1}}^{t_{2}} f_{0}\left(t, y_{k}(t), z_{k}(t), w_{k}(t)\right) d t \\
& =\inf _{\Gamma_{0}(x)} \frac{\lim }{k \rightarrow \infty} I_{0}\left(y_{k}, z_{k}, w_{k}\right),
\end{aligned}
$$

where $\Gamma_{0}(x)$ denotes the class of all sequences $\left(x_{k}, w_{k}\right)_{k \in N}$ in $\Omega_{0}$ such that (a) $x_{k}=\left(y_{k}, z_{k}\right) \in \mathrm{ACg}, k \in N$; (b) $y_{k} \rightarrow y$ uniformly and $z_{k} \rightarrow z$ pointwise a.e. in $\left[t_{1}, t_{2}\right]$. If $\Gamma_{0}(x)=\emptyset$ we take $\mathscr{I}_{0}(x)=+\infty$. The class $\Omega_{0}$ is said to be closed if it has the following property (c): If $\left(y_{k}, z_{k}, w_{k}\right)_{k \in N}$ is a sequence of admissible systems, all in $\Omega_{0}$, satisfying (a) and (b), and if there exists a measurable $w$ such that $(y, z, w)$ is an admissible system, then $(y, z, w)$ belongs to $\Omega_{0}$.

Note that, if the problem of minimizing the functional above involves given initial or terminal values for $x$, say $x\left(t_{1}\right) \in B_{1}, x\left(t_{2}\right) \in B_{2}$, then we will proceed as illustrated in Remark 2 of Section 3.

It is well known (see [5], Section 1.13) that the problem of optimal control described above can be deparametrized, and essentially reduced to a problem of calculus of variations as discussed in Section 3. For every $(t, x) \in A$ let $Q(t, x)$ denote the set

$$
Q(t, x)=\left\{\zeta \in R^{n}: \zeta=f(t, x, w), w \in U(t, x)\right\}
$$

and take

$$
M=\left\{(t, x, \zeta) \in R^{2 n+1}:(t, x) \in A, \zeta \in Q(t, x)\right\}
$$


Let $F_{0}(t, x, \zeta)$ denote the scalar function defined on $M$ by taking

$$
F_{0}(t, x, \zeta)=\inf \left\{z^{0} \in R ; z^{0} \geqq f_{0}(t, x, w), \zeta=f(t, x, w), w \in U(t, x)\right\} .
$$

If for some $\zeta$ the set in brackets is empty, we take $F_{0}=+\infty$. If in (1) inf is actually a minimum for all $(t, x, \zeta) \in M$, then we may replace the problem of optimal control with the problem of the calculus of variations studied in Section 3, concerning the integral functional $\mathscr{I}$ relative to the integrand $F_{0}$, with constraints $(t, x(t)) \in A, x^{\prime}(t) \in Q(t, x(t))$, a.e. in $\left[t_{1}, t_{2}\right]$, and where $x=(y, z), y \in \mathrm{ACg}$, $z \in$ BVC. We will apply Theorem 2 of Section 3 to the present problem of the calculus of variations. Of course, we shall assume that the sets $Q(t, x)$ are nonempty and convex and that the scalar function $F_{0}(t, x, \zeta)$ is lower semicontinuous in $(t, x, \zeta)$ and convex in $\zeta$. Moreover, once we have a solution $x=(y, z)$ of the deparametrized problem, or problem of the calculus of variations, we shall need to know that there exists some measurable function $w(t)$, or $w:\left[t_{1}, t_{2}\right] \rightarrow R^{m}$ such that

$$
\begin{gathered}
w(t) \in U(t, x(t)), f_{0}(t, x(t), w(t))=F_{0}\left(t, x(t), x^{\prime}(t)\right), f(t, x(t), w(t))=x^{\prime}(t), \\
\text { a.e. in }\left[t_{1}, t_{2}\right] .
\end{gathered}
$$

This is a consequence of the implicit function theorems. For instance, if $f_{0}$ and $f$ are continuous on the closed set $M_{0}$, then the existence of a measurable $w(t)$ satisfying (2) follows from the McShane-Warfield implicit function theorem ([5], Theorem 8.2.iii). In [5], Sections 8.2., 8.3, a great many situations are depicted for which some implicit function theorem applies. Concerning the $n$-vector function $f(t, x, w)=\left(f_{1}, \ldots, f_{n}\right)$, we write $\tilde{f}_{1}=\left(f_{1}, \ldots, f_{\alpha}\right)$ and $\tilde{f}_{2}=\left(f_{a+1}, \ldots, f_{n}\right)$. We shall need the following alternative assumptions:

$\left(\mathrm{g}_{1}\right)$ There is a scalar function $\phi(\zeta), 0 \leqq \zeta<+\infty$, or $\phi: R_{0}^{+} \rightarrow R$ bounded below, such that $\phi(\zeta) / \zeta \rightarrow+\infty$, as $\zeta \rightarrow+\infty$, and $f_{0}(t, x, w) \geqq \phi\left(\left|\tilde{f_{1}}(t, x, w)\right|\right)$ for all $(t, x, w) \in M_{0}$.

$\left(\mathrm{g}_{2}\right)$ For every $\varepsilon>0$ there is a summable scalar function $\psi_{\varepsilon}(t) \geqq 0$ such that $\left|\tilde{f_{1}}(t, x, w)\right| \leqq \psi_{\varepsilon}(t)+\varepsilon f_{0}(t, x, w)$ for all $(t, x, w) \in M_{0}$.

$\left(\mathrm{g}_{3}\right)$ For any $\alpha$-vector $p \in R^{\alpha}$ there is a summable scalar function $\phi_{p}(t) \geqq 0$, such that $f_{0}(t, x, w) \geqq\left\langle p, \tilde{f}_{1}(t, x, w)\right\rangle-\phi_{p}(t)$, for all $(t, x, w) \in M_{0}$.

Note that, under condition $\left(\mathrm{g}_{1}\right)$, certainly $\phi(\zeta) \geqq \lambda$ for some real constant $\lambda$, and then $f_{0}(t, x, w) \geqq \phi\left(\left|\tilde{f}_{1}(t, x, w)\right|\right) \geqq \lambda$ for all $(t, x, w) \in M_{0}$. Under condition $\left(\mathrm{g}_{2}\right)$ and $\varepsilon=1$, we have $\left|f_{1}\right| \leqq \psi_{1}(t)+f_{0}(t, x, w)$; hence $f_{0}(t, x, w) \geqq$ $-\psi_{1}(t)$, a summable function. Under condition $\left(\mathrm{g}_{3}\right)$ and $p=0$. we have $f_{0}(t, x, w)$ $\geqq-\phi_{0}(t)$, a summable function.

Theorem 3 (An existence theorem for problems of Optimal Control). Let $1 \leqq \alpha \leqq n-1$, and assume that (i) $A$ is compact and $M_{0}$ is closed; (ii) the sets $\tilde{Q}(t, x)$ are closed, convex and satisfy property $(\mathrm{Q})$ with respect to $(t, x)$ at every point $(t, x)$ of $A$ (with the exception perhaps of a set of points whose $t$-coordinate lies on a set of measure zero on the t-axis); (iii) the functions $f$ and $f_{0}$ are continuous and satisfy one of the growth conditions $\left(\mathrm{g}_{1}\right),\left(\mathrm{g}_{2}\right),\left(\mathrm{g}_{3}\right)$. Also we assume that 
the class $\Omega_{0}$ is nonempty and closed, and (iv) there is a constant $W_{0}$ such that for every element $x=(y, z) \in \Omega_{0} \cap \mathrm{ACg}$, then $V^{*}(z) \leqq W_{0}$. Then the functional $\mathscr{I}_{0}$ has an absolute minimum $x=(y, z)$ in $\Omega_{0}$.

For $\alpha=0$, then $x=z$, the requirements $\left(\mathrm{g}_{1}\right)$ or $\left(\mathrm{g}_{2}\right)$ or $\left(\mathrm{g}_{3}\right)$ do not apply, yet the conclusion is still valid if we know that (iii') there is a summable scalar function $\lambda:\left[t_{1}, t_{2}\right] \rightarrow R$ such that $f_{0}(t, x, w) \geqq \lambda(t)$, for all $(t, x, w) \in M_{0}$.

For $\alpha=n$, then $x=y, \Omega_{0}$ is a nonempty and closed class of $\mathrm{ACg}$ functions $y(t)=\left(y^{1}, \ldots, y^{n}\right), t \in\left[t_{1}, t_{2}\right]$. condition (iv) does not apply, and the problem reduces essentially to those discussed in Theorems 11.4.i. and ii of [5].

Statement 3 is a corollary of Theorem 2 .

Note that for $0 \leqq \alpha \leqq n-1$, if (iv') there are scalar functions $\psi_{i} \in L_{1}\left(\left[t_{1}, t_{2}\right]\right.$, $i=\alpha+1, \ldots, n$, such that $(t, y, z, u, v) \in M_{0}$ implies $v^{i} \geqq \psi_{i}(t)$ a.e. in $\left[t_{1}, t_{2}\right]$, then (iv) certainly holds.

Note that, for $1 \leqq \alpha \leqq n-1$, the sets $\tilde{Q}(t, x)$ are closed and convex, $\left(g_{1}\right)$ holds, and if $\left(\mathrm{iv}^{\prime \prime}\right)$ there exist constants $L_{i}$ such that a.e. in $\left[t_{1}, t_{2}\right],(t, y, z, u, v) \in M_{0}$ implies $v^{i} \geqq L_{i}, i=\alpha+1, \ldots, n$, then both (ii) and (iv) hold.

See also Remarks 6 and $6^{\prime}$.

Acknowledgment. This research was partially supported by the G.N.A.F.A.-C.N.R.

\section{References}

1. M. Boni, Variazione generalizzata con peso e quasi additività, Atti Sem. Mat. Fis. Univ. Modena 25 (1976), 195-210.

2. M. Boni \& P. Brandi, Variazione classica e generalizzata con peso, Atti Sem. Mat. Fis. Univ. Modena 23 (1974), 286-307.

3. D. Candeloro \& P. Pucci, L'integrale di Burkill-Cesari come integrale del Calcolo delle Variazioni, Boll. Un. Mat. Ital. (5) 18-B (1981), 1-24.

4. L. Cesari, Sulle funzioni a variazione limitata, Ann. Scuola Norm. Sup. Pisa 5 (1936), 299-313.

5. L. Cesari, Optimization-Theory and Applications, Springer Verlag (1983).

6. L. Cesari, P. BRANDI \& A. SAlvadorI, (a) Discontinuos solutions in problems of optimization, Ann. Scuola Norm. Sup. Pisa, to appear. - (b) Existence theorems for multiple integrals of the calculus of variations for discontinuous solutions, Annali Mat. pura appl., to appear.

7. L. CESARI \& P. PUCCI, An elementary proof of an equivalence theorem relevant in the theory of optimization, J. Optimization Theor. Appl. 46 (1985), 215-226.

8. E. ConwaY \& J. SMOLleR, Global solutions of the Cauchy problem for quasi-linear first-order equations in several space variables, Comm. Pure Appl. Math. 19 (1966), 95-105.

9. C. M. DAFERMOS, Generalized characteristics and the structure of solutions of hyperbolic conservation laws, Indiana Univ. Math. J. 26 (1977), 1097-1119.

10. R. J. DI PERnA, Singularities of solutions of nonlinear hyperbolic systems of conservation laws, Arch. Rational Mech. Anal. 60 (1975), 75-100.

11. C. GofFMaN, Lower semicontinuity and area functionals. I. The non parametric case, Rend. Circ. Mat. Palermo 2 (2) (1953), 203-235.

12. K. KRICKEBERG, Distributionen, Funktionen beschränkter Variation und Lebesguescher Inhalt nichtparametrischer Flächen, Ann. Mat. Pura Appl. 44 (1957), 105-133. 
13. A. SAlvadori, Sulla convergenza in lunghezza con peso per una successione di curve parametriche, Rend. Circ. Mat. Palermo 36 (2) (1977), 195-228.

14. J. SERRIN, On the definition and properties of certain variational integrals, Trans. Amer. Math. Soc. 101 (1961), 139-167.

15. C. VINTI, L'integrale di Weierstrass-Burkill, Sem. Mat. Fis. Univ. Modena 18 (196), 295-316.

16. A. L. VOLPERT, The spaces BV and quasilinear equations, Mat. Sb. 73 (1967), 225-267.

University of Michigan

Ann Arbor

and

Università degli Studi

Perugia

(Received April 25, 1986) 